\title{
Galactic bulges: the importance of early formation scenarios vs. secular evolution
}

\author{
Marja K. Seidel ${ }^{1,2}$, R. Cacho ${ }^{3}$, T. Ruiz-Lara ${ }^{4,5}$, J. Falcón-Barroso ${ }^{1,2}$, \\ I. Pérez ${ }^{4,5}$, P. Sánchez-Blázquez ${ }^{6}$, F. P. A. Vogt $^{7}$, M. Ness ${ }^{8}$, \\ K. Freeman ${ }^{7}$ and S. Aniyan ${ }^{7,9}$ \\ ${ }^{1}$ Instituto de Astrofísica de Canarias, E-38200 La Laguna, Tenerife, Spain \\ ${ }^{2}$ Departamento de Astrofísica, Universidad de La Laguna, E-38205 La Laguna, Tenerife, Spain \\ email: mseidel@iac.es \\ ${ }^{3}$ Dpto. de Astrofísica y CC. de la Atmósfera, Universidad Complutense de Madrid, Spain \\ ${ }^{4}$ Dpto. de Física Teórica y del Cosmos, Universidad de Granada, Facultad de Ciencias \\ (Edificio Mecenas), 18071 Granada, Spain \\ ${ }^{5}$ Instituto Universitario Carlos I de Física Teórica y Computacional, Universidad de Granada, \\ 18071 Granada, Spain \\ ${ }^{6}$ Dpto. de Física Teórica, Universidad Autónoma de Madrid, E-28049 Cantoblanco, Spain \\ ${ }^{7}$ Research School of Astronomy and Astrophysics, Australian National University, Canberra, \\ ACT 2611, Australia \\ ${ }^{8}$ Max-Planck-Institut für Astronomie, Königstuhl 17, D-69117 Heidelberg, Germany \\ ${ }^{9}$ European Southern Observatory, Karl-Schwarzschild-Str. 2, 85748 Garching, Germany
}

\begin{abstract}
We study the stellar content of three galactic bulges with the high resolution gratings $(\mathrm{R}=7000)$ of the WiFeS integral field unit in order to better understand their formation and evolution. In all cases we find that at least $50 \%$ of the stellar mass already existed 12 Gyrs ago, more than currently predicted by simulations. A younger component (age between $\sim 1$ to $\sim 8$ Gyrs) is also prominent and its present day distribution seems to be much more affected by morphological structures, especially bars, than the older one. This in-depth analysis supports the notion of increasing complexity in bulges which cannot be achieved by mergers alone, but requires a non-negligible contribution from secular evolution.
\end{abstract}

Keywords. galaxies: individual (NGC 5701, NGC 6753, NGC 7552), galaxies: bulges, galaxies: evolution, galaxies: kinematics and dynamics, galaxies: stellar content, techniques: spectroscopic

\section{Introduction}

Galactic bulges, considered as a deviation from the exponential profile in the centres of galaxies, hold crucial clues to refine our understanding of galaxy formation and evolution. Yet, the details of the interplay of collapse, mergers and secular processes are still not fully understood. The review by Kormendy \& Kennicutt (2004) (and references therein) summarizes the two main bulge formation mechanisms: i) merger-driven and ii) secularly evolved. The advances in instrumentation, spectral libraries (e.g., MILES, Sánchez-Blázquez et al. 2006), and stellar population (SP) studies via full-spectral fitting techniques (e.g. STECKMAP: Ocvirk et al. 2006) now allow us to characterize the stellar content of galaxies on a spatially resolved basis, and test the different bulge formation mechanisms. To that end, we conducted a pilot study using the WiFeS integral field unit (and its high resolution mode; $\mathrm{R}=7000$ ) to link the stellar kinematics and SP parameters (and their radial distribution). The three bulges analyzed are NGC 5701, NGC 6753 and NGC 7552 and their unsharp masks are shown in Fig. 1 along with their kinematics. The high quality of our data allows us to characterize the $2 \mathrm{D}$ distribution of different stellar populations (i.e. young, intermediate, and old, see Fig. 1, middle and bottom row). 


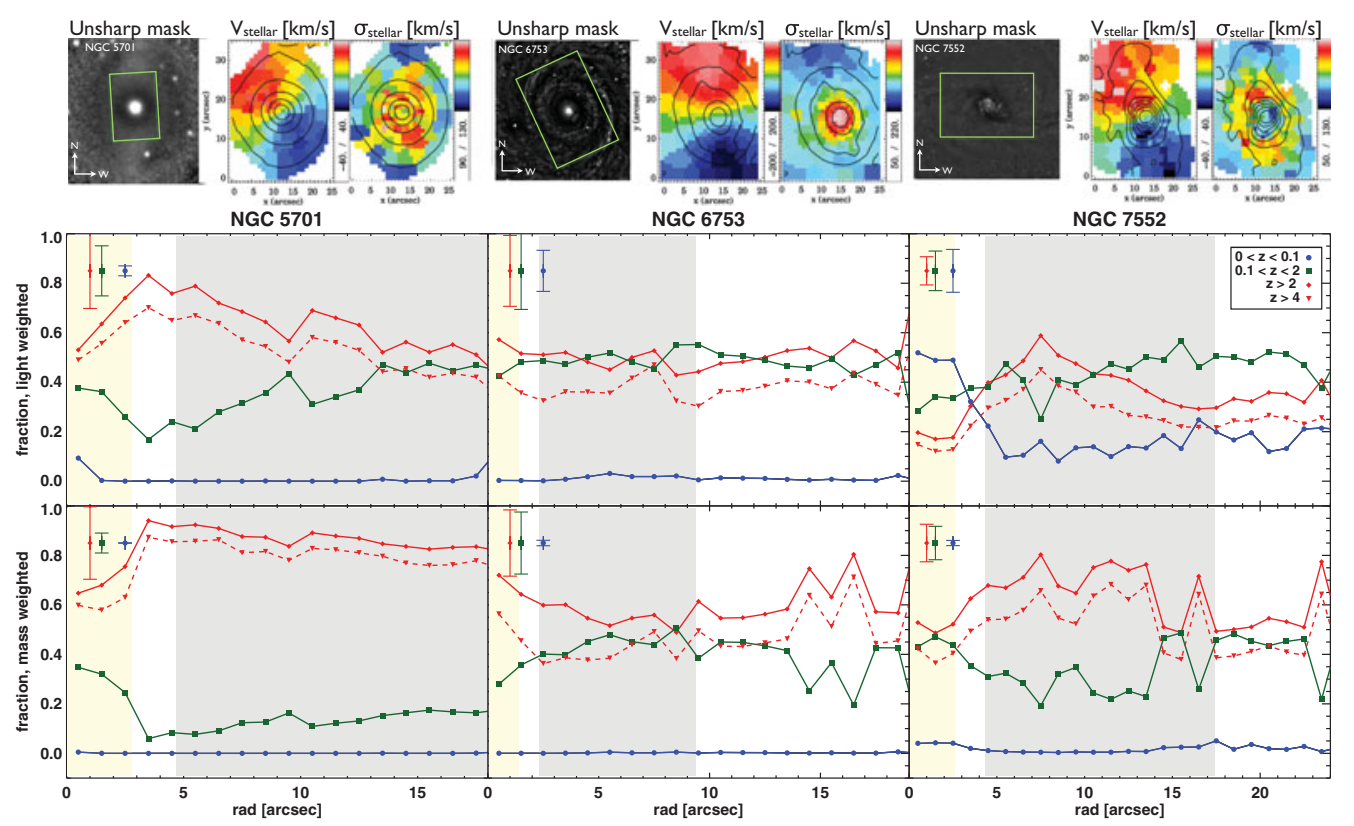

Figure 1. Top row: Unsharp masks (Spitzer image for NGC 5701, HST WFPC2 F814W filter for NGC 6753 and NGC 7552$)$ with the WiFeS FoV $(25 \times 38$ arcsec) in green, stellar velocity and stellar velocity dispersion map. Below: Fractions of young (blue dots), intermediate (green squares) and old SP (red rhombus) as a function of radius. Top left corner: corresponding uncertainties. Middle row: L-weighted and Bottom row: M-weighted results. Shaded regions show the central $(<0.3 \mathrm{kpc})$ and inner parts $(0.5 \mathrm{kpc}<\mathrm{r}<2 \mathrm{kpc})$.

\section{Conclusions: Old bulge structures rejuvenated by bars}

Our main conclusions are threefold (as illustrated in Fig. 1):

- The stellar velocity dispersion is higher in regions where the light of the old population is dominant

- The fraction of the mass of the old stellar population (existing already at $\mathrm{z} \sim 2$ ) is $50-80 \%$ in the inner $2 \mathrm{kpc}$ of all bulges, hinting towards high star formation (SF) during the collapse in the early cosmic web

- The barred galaxies (NGC 5701, NGC 7552) show a significant amount of light of the young SP and mass of the intermediate SP in the centres, likely indicating the influence of the bars driving necessary material towards the centre to fuel this second SF period.

These conclusions are based on a sample that cannot be regarded as representative. But as all our bulges show consistent features albeit their different nature (mass, morphology), it hints towards a common evolution. More details in Seidel et al. (2014, submitted).

\section{Acknowledgements}

This research is supported by the Spanish Ministry of Economy and Competitiveness (AYA2010-21322-C03-02 \& AYA2009-11137) and of Science and Innovation (AYA201124728 \& Consolider-Ingenio CSD2010-00064) and by the Junta de Andalucía (FQM-108).

\section{References}

Kormendy J. \& Kennicutt, Jr., R. C. 2004, Annual Review of Astronomy \& Astrophysics, 42, 603-683

Sánchez-Blázquez, P., Peletier, R. F., Jiménez-Vicente, J., Cardiel, N., Cenarro, A. J., Falcón-Barroso, J., Gorgas, J., Selam, S., \& Vazdekis, A. 2006, MNRAS, 371, 703-718

Ocvirk, P. , Pichon, C., Lançon, A., \& Thiébaut, E. 2006, 365, 74-84 